\title{
Assembling Hybrid Energetic Materials with Controllable Interfacial Microstructure by Electrospray
}

Lihong Chen ${ }^{a, b, c}, \quad$ Chengbo Ru ${ }^{b, c, *}$, Hongguo Zhang ${ }^{b, c}$, Yanchun Zhang ${ }^{b, c}$, Zhiwei Chi ${ }^{b}$, Haoyuan Wang ${ }^{b}$, Gang $\mathrm{Li}^{a}$

${ }^{a}$ Fire \& Explosion Protection Laboratory, Northeastern University, Shenyang 110819,

China

${ }^{\mathrm{b}}$ College of Forensic Science, Criminal Investigation Police University of China,

Shenyang 110035, China.

${ }^{c}$ Key Laboratory of Impression Evidence Examination and Identification Technology, Ministry of Public

Security, Shenyang 110035, China

"Corresponding author E-mail: ruchengbo@163.com; ruchengbo2012@gmail.com. 
Table S1. Formula of samples, where the $\varphi$ of nano $\mathrm{Al} / \mathrm{CuO}$ was fixed at 1.4 (UMMechanical Mixing, ES-Electrospray).

\begin{tabular}{lll}
\hline Solvents & abbreviation & Existence of CL-20 in solvents \\
\hline n-Propanol/Diethyl Ether & NPA/DEE & Nanoparticles and solute (saturated solution) \\
Ethyl Acetate & EAC & Solute (dilute solution) \\
Acetone & DMK & solute (dilute solution) \\
Ethyl Acetate/Acetone & EAC/DMK & Solute (dilute solution) \\
Ethanol/Diethyl Ether & EA/DEE & solute (nearly saturated solution) \\
Ethyl Acetate/Acetone & EAC/DMK & solute (dilute solution) \\
\hline
\end{tabular}

Table S2. Physic properties and $\varepsilon$-CL-20 solubility of differnent solvents

\begin{tabular}{|c|c|c|c|c|c|c|}
\hline Solvents & $\begin{array}{c}\text { Diethyl } \\
\text { Ether }\end{array}$ & Hexane & Acetone & Ethanol & $\begin{array}{c}\text { Ethyl } \\
\text { Acetate }\end{array}$ & $\begin{array}{c}\text { 1-Propan } \\
\text { ol }\end{array}$ \\
\hline $\begin{array}{l}\text { Solubility of CL-20 } \\
\left(\mathrm{mg} / \mathrm{mL} \text {, at } 20^{\circ} \mathrm{C}\right)\end{array}$ & I & insoluble & 748 & 5.03 & 461.2 & 1.59 \\
\hline $\begin{array}{c}\text { Viscosity } \\
\left(\mathrm{cP} \text {, at } 25^{\circ} \mathrm{C}\right)\end{array}$ & 0.22 & 0.307 & 0.31 & 1.07 & 0.42 & 2.26 \\
\hline Electrical & $3.7 \times 10^{-13}$ & I & $5.8 \times 10^{-}$ & $1.35 \times 10^{-}$ & $3.0 \times 10^{-9}$ & $9.17 \times 10^{-9}$ \\
\hline
\end{tabular}


conductivity $(\mathrm{S} / \mathrm{m})$

Vapor pressure

$\left(\mathrm{kPa}\right.$, at $\left.25^{\circ} \mathrm{C}\right)$

Surface tension

16.7

$(\mathrm{mN} / \mathrm{m})$

58.7

$17 \quad 24.0$

5.9

9.7

2.0
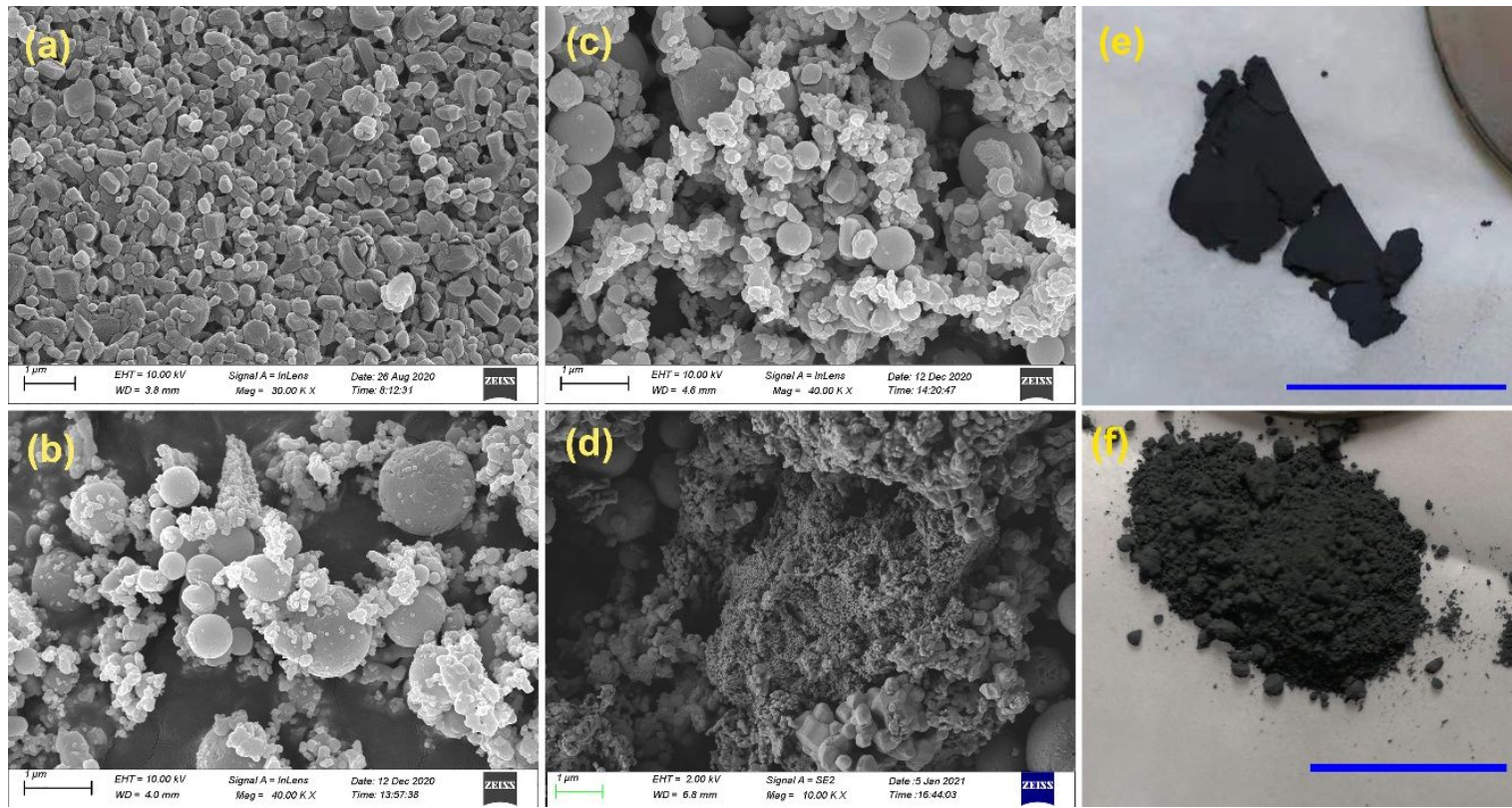

Figure S1. SEM images of HEM prepared by ultrasonic mixing, (a) pristine CL-20, (b)

$\mathrm{Al} / \mathrm{CuO}(\mathrm{UM}, \quad$ hexane), (c) $\mathrm{Al} / \mathrm{CuO} / \mathrm{CL}-20 \quad$ (UM, hexane), and (d) $\mathrm{Al} / \mathrm{CuO} / \mathrm{NC} / \mathrm{CL}-20(\mathrm{UM}$, acetone), (e) clay-like HEMs(ES EAC) and (f) granular HEMs(ES DMK). The scale bars in (e) and (f) are $15 \mathrm{~mm}$. 


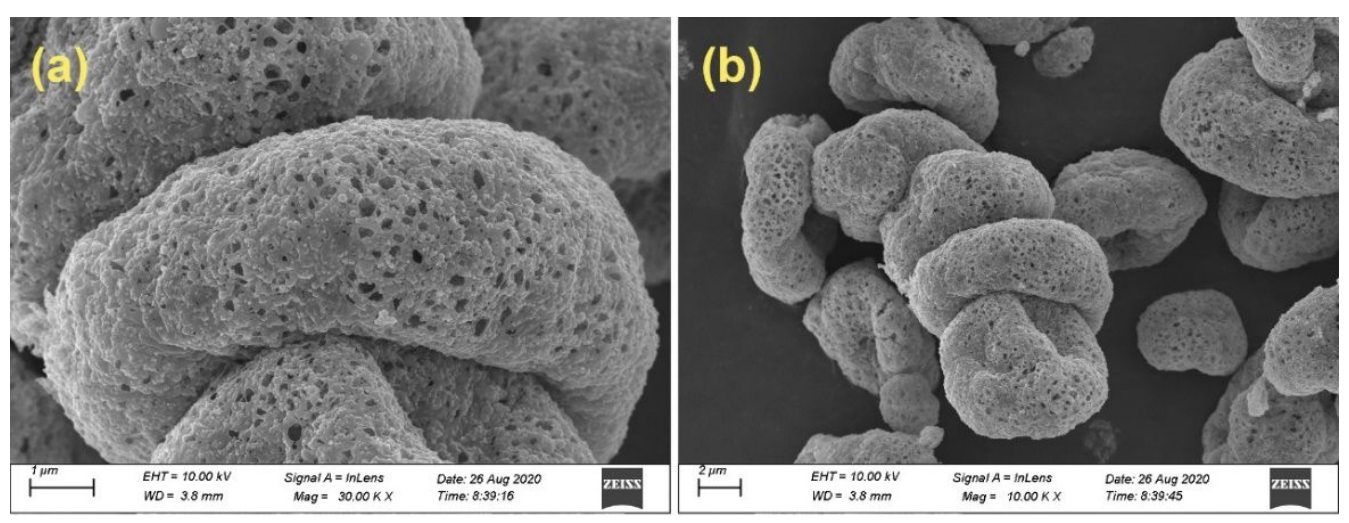

Figure S2. SEM images of assembled HEMs from pure ethyl acetate by electrospray at different magnification, (a) 30k and (b) 10k, no NC fiber is observed (at $25^{\circ} \mathrm{C}$ ).

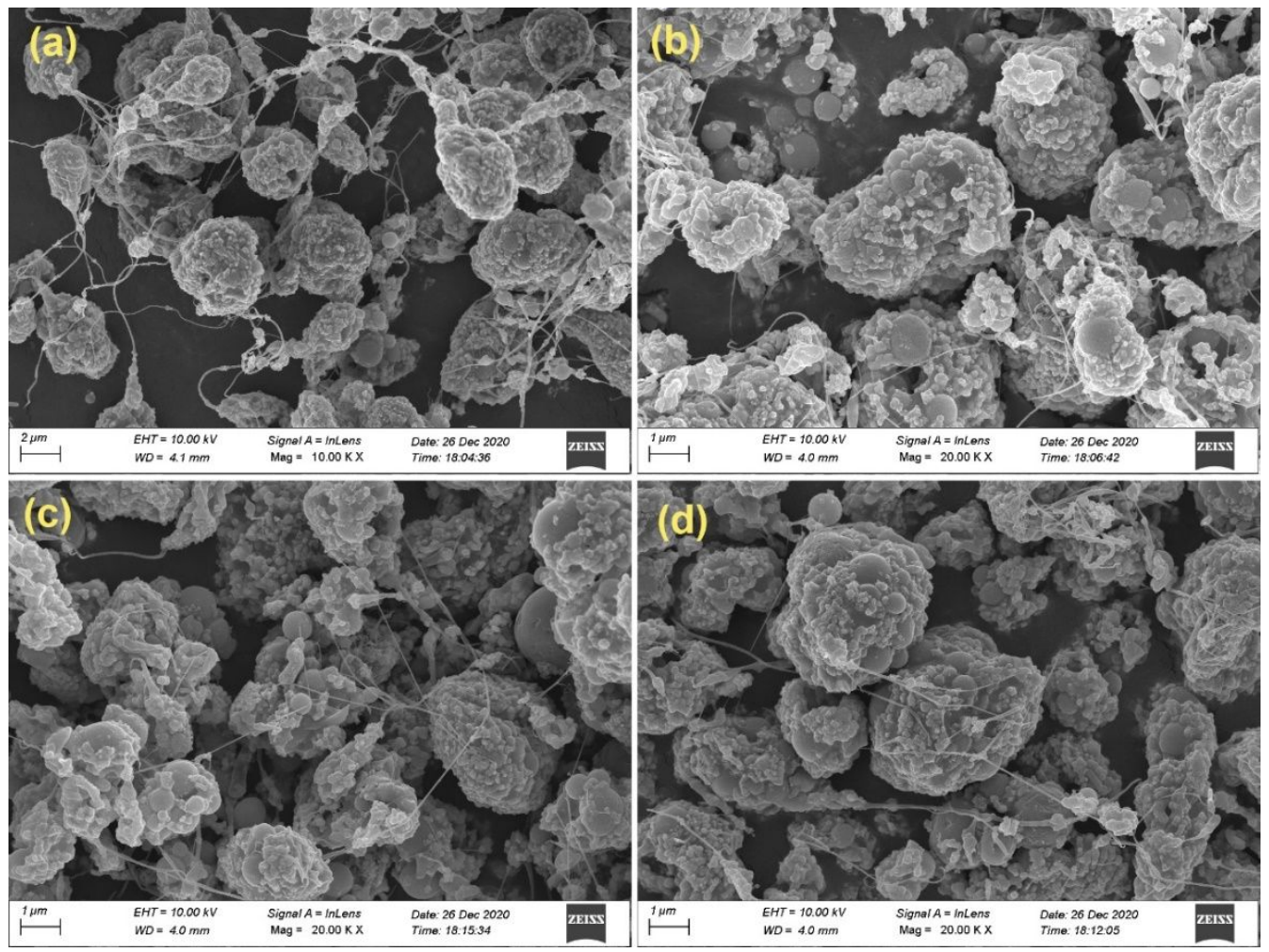


Figure S3. SEM images of as-prepared HEM obtained from the mixtures of acetone and ethyl acetate, (a) VDMK/VEAC=1:3, (b) VDMK/VEAC=2:1, (c) VDMK/VEAC=3:1, and (d) VDMK/VEAC $=5: 1$.

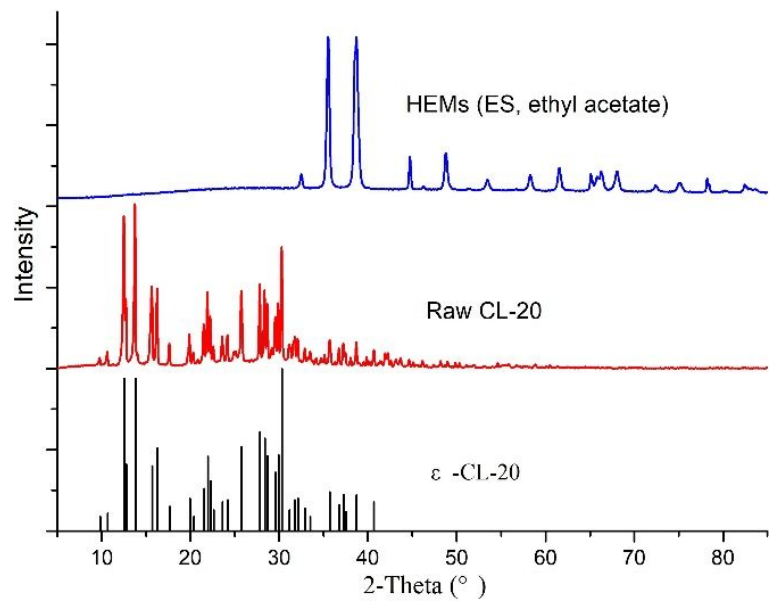

Figure S4. XRD patterns of JCPDS No.50-2045 ( $\varepsilon$-CL-20), raw CL-20, and HEMs (ES, ethyl acetate). No characteristic peak of CL-20 appears in the pattern of HEMs.

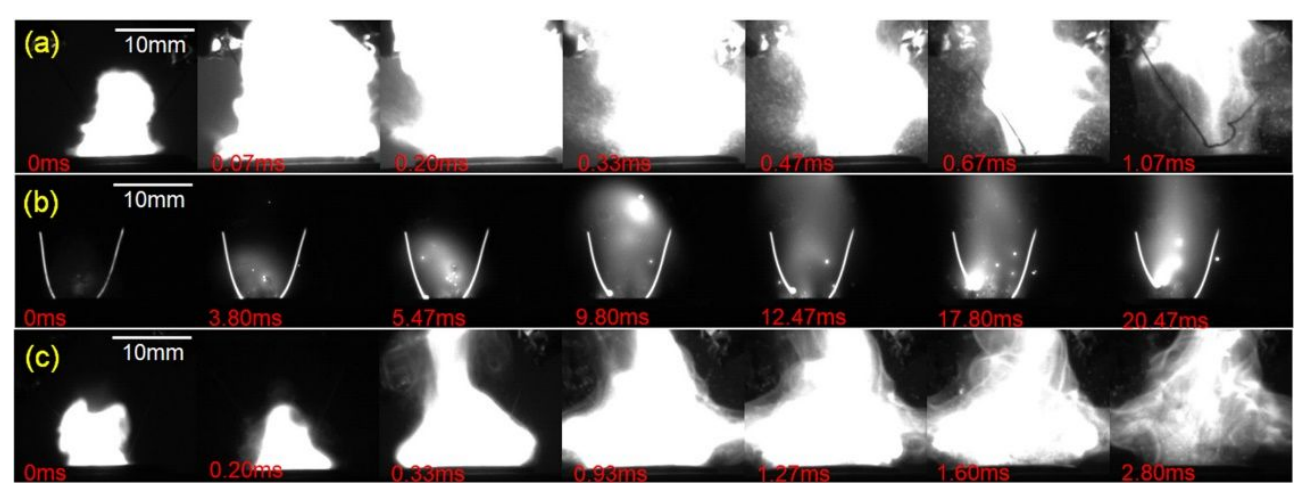

Figure S5. Combustion processes recorded by a high speed camera at $15000 \mathrm{fps}$, minimum aperture and $15 \mu \mathrm{s}$ exposure, of (a) $\mathrm{Al} / \mathrm{CuO}$ (UM), (b) CL-20 and (c) 
$\mathrm{Al} / \mathrm{CuO} / \mathrm{CL}-20$ (UM). The flame brightness of raw CL-20 is too low to be discern, so the

c exposure time and aperture were adjusted to larger aperture ( 4 times larger area than

the minimum aperture) and $50 \mu$ s exposure.

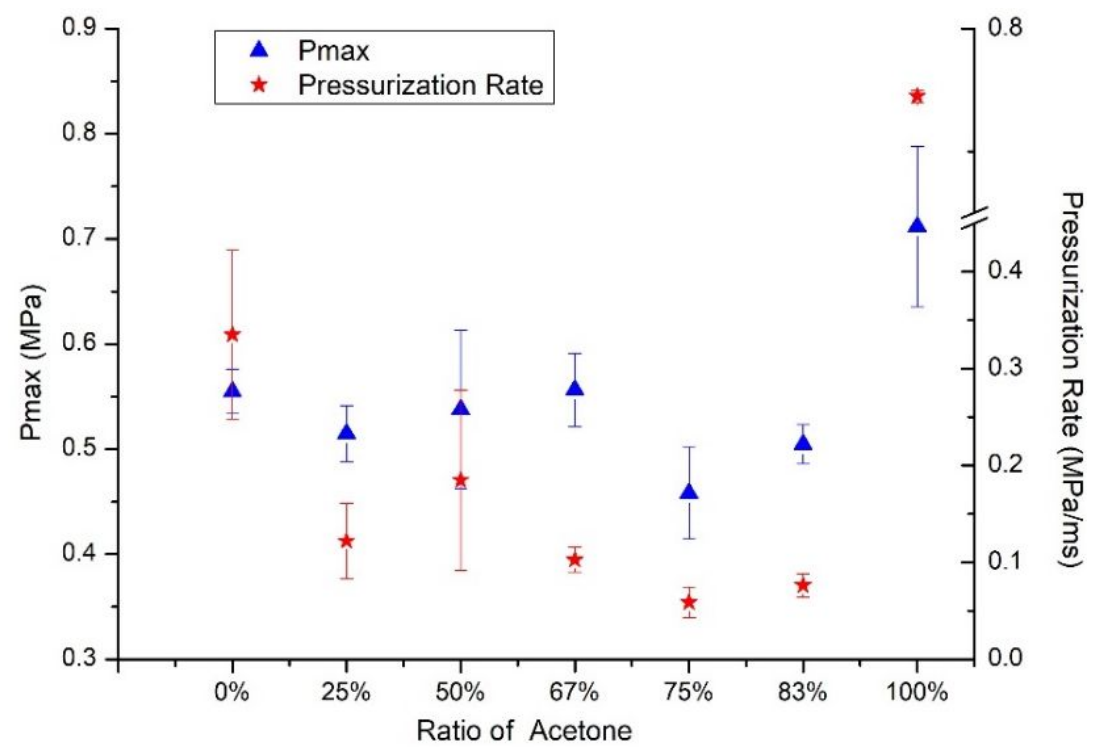

Figure S6. Pressurization characteristics of as-prepared Al/CuO/NC/CL-20 (ES) with different mixtures of acetone and ethyl acetate. 


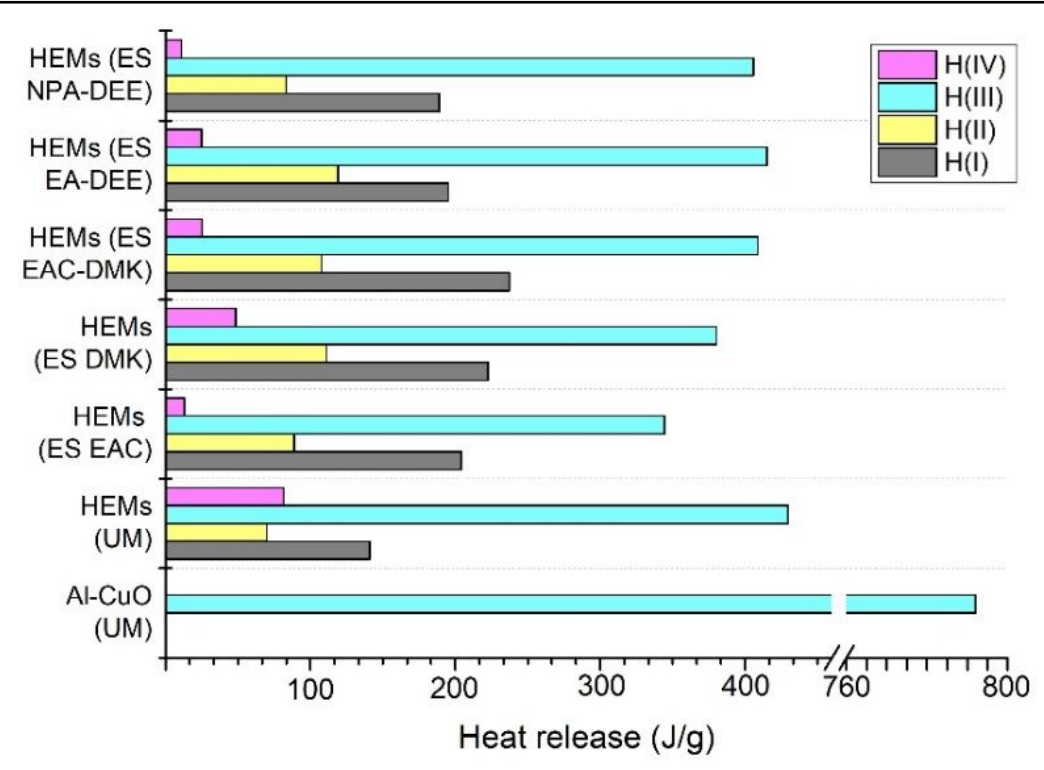

Figure S7. Heat release of $\mathrm{HEMs}$ and $\mathrm{Al} / \mathrm{CuO}$ in different exothermic stages.

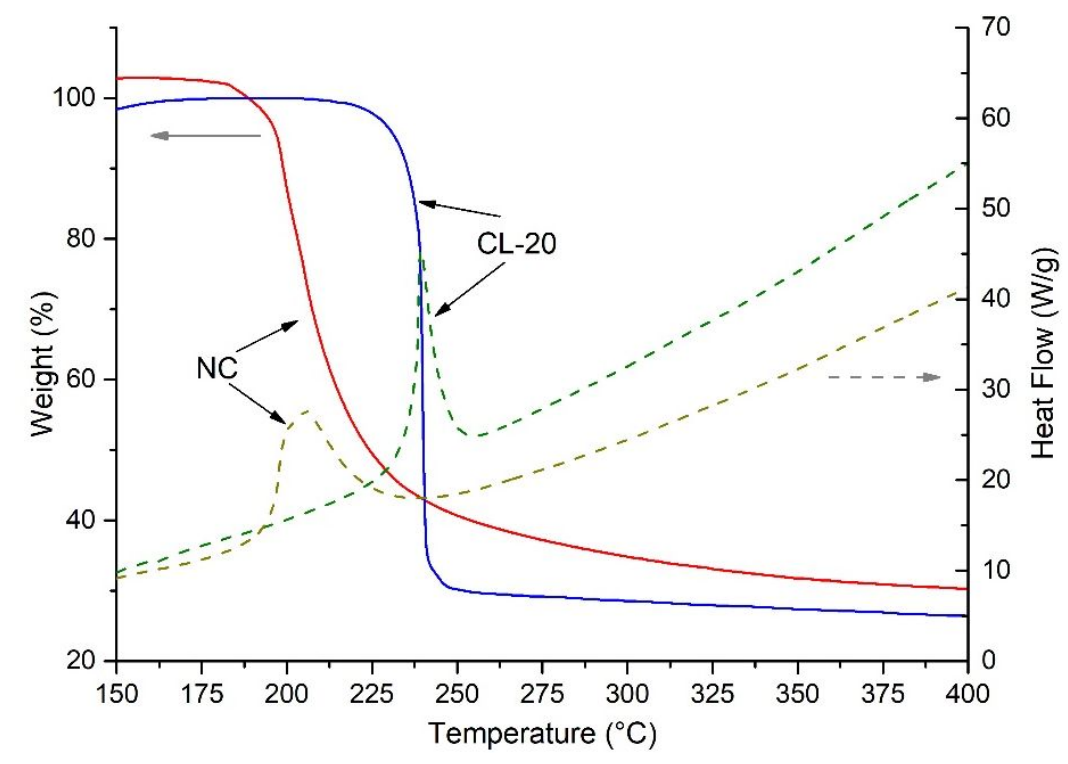

Figure S8. TG and DSC curves of CL-20 and NC 


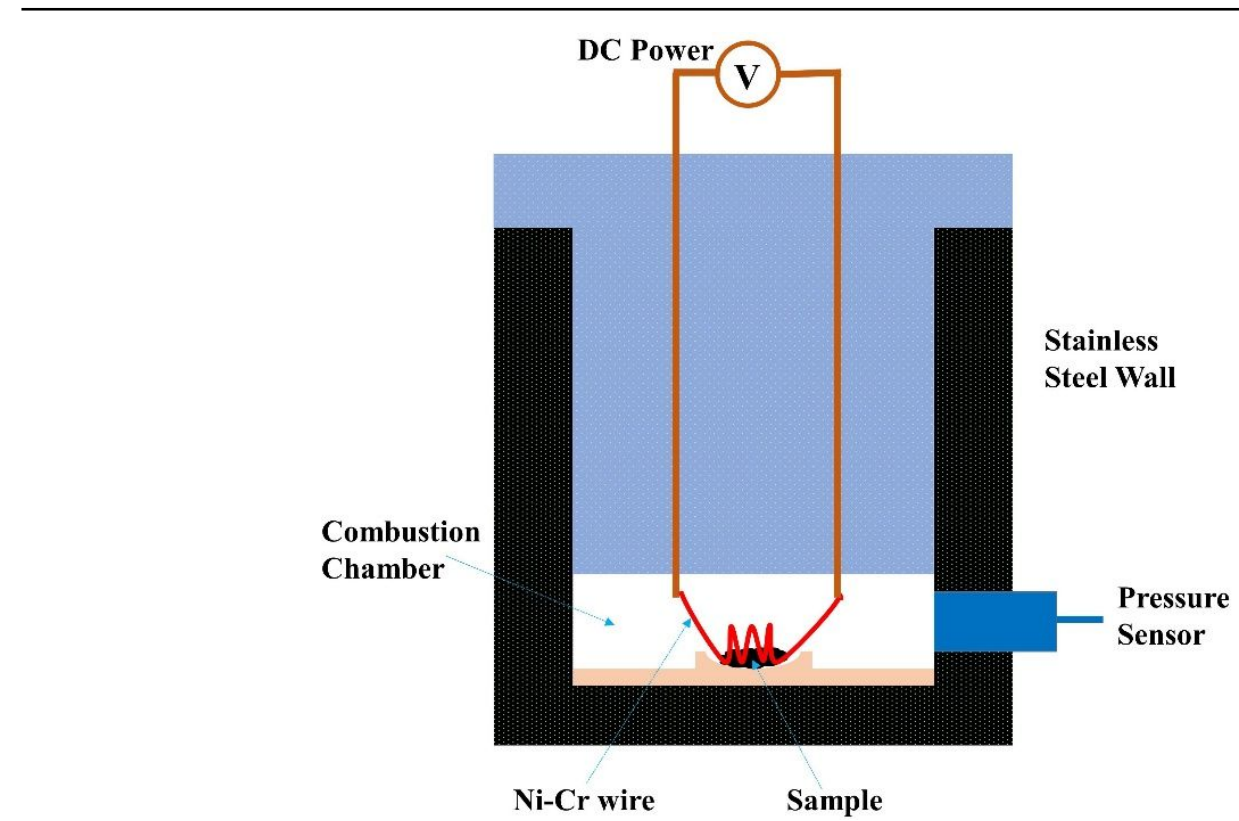

Figure S9. Schematic diagram of pressure cell 
Table S3 Exothermic peak temperatures of samples at different heating rates.

\begin{tabular}{|c|c|c|c|c|}
\hline Samples & Heating rate $(K / \mathrm{min})$ & $T_{p I}\left({ }^{\circ} \mathrm{C}\right)$ & $T_{p I I}\left({ }^{\circ} \mathrm{C}\right)$ & $T_{p I I I}\left({ }^{\circ} \mathrm{C}\right)$ \\
\hline \multirow{3}{*}{$N C$} & 5 & 189.6 & & \\
\hline & 10 & 193.6 & & \\
\hline & 20 & 198.0 & & \\
\hline \multirow{3}{*}{$C L-20$} & 5 & 226.1 & & \\
\hline & 10 & 232.7 & & \\
\hline & 20 & 236.9 & & \\
\hline \multirow{3}{*}{$\begin{array}{l}\mathrm{A} / / \mathrm{CuO} \\
(U M)\end{array}$} & 5 & & & 575.6 \\
\hline & 10 & & & 587.0 \\
\hline & 20 & & & 600.2 \\
\hline \multirow{3}{*}{$\begin{array}{l}A / / C u O / C L-20 \\
(U M)\end{array}$} & 5 & 218.2 & 325.3 & 544.6 \\
\hline & 10 & 226.7 & 337.0 & 559.4 \\
\hline & 20 & 236.7 & 348.1 & 576.2 \\
\hline \multirow{3}{*}{$\begin{array}{l}H E M S \\
(E S E A C)\end{array}$} & 5 & 215.3 & 386.2 & 604 \\
\hline & 10 & 223.4 & 401.6 & 628.1 \\
\hline & 20 & 231.4 & 419.8 & 656 \\
\hline \multirow{3}{*}{$\begin{array}{l}\text { HEMS } \\
(E S D M K)\end{array}$} & 5 & 221.2 & 388.7 & 585.5 \\
\hline & 10 & 228.8 & 399.7 & 629.7 \\
\hline & 20 & 234.7 & 413.4 & 658.5 \\
\hline \multirow{3}{*}{$\begin{array}{l}H E M S \\
(E S E A / D E E)\end{array}$} & 5 & 220.3 & 395.6 & 589.2 \\
\hline & 10 & 227.5 & 406.4 & 593.5 \\
\hline & 20 & 235.2 & 423.8 & 627.5 \\
\hline \multirow{3}{*}{$\begin{array}{l}\text { HEMS } \\
(E S N P A / D E E)\end{array}$} & 5 & 215.2 & 390.7 & 608.5 \\
\hline & 10 & 224.6 & 402.4 & 633.9 \\
\hline & 20 & 233.1 & 422.2 & 658.2 \\
\hline
\end{tabular}

